EPJ manuscript No.

(will be inserted by the editor)

\title{
Melting and evaporation transitions in small Al clusters: canonical Monte-Carlo simulations
}

\author{
Ralph Werner \\ Institut für Theorie der Kondensierten Materie, Universität Karlsruhe, D-76128 Karlsruhe, Germany
}

Version: September 23, 2018

\begin{abstract}
A dimer of bound atoms cannot melt, only dissociate. Bulk metals show a well defined first order transition between their solid and liquid phases. The appearance of the melting transition is explored for increasing clusters sizes via the signatures in the specific heat and the root mean square of the bond lengths $\delta_{\mathrm{B}}$ (Berry parameter) by means of Monte-Carlo simulations of Al clusters modelled by Gupta potentials. Clear signatures of a melting transition appear for $N \sim 6$ atoms. Closed-shell effects are shown for clusters with up to 56 atoms. The melting transition is compared in detail with the dissociation transition, which induces a second and possibly much larger local maximum in the specific heat at higher temperatures. Larger clusters are shown to fragment into dimers and trimers, which in turn dissociate at higher temperatures.
\end{abstract}

PACS. 61.46. $+\mathrm{w}$ Nanoscale materials: clusters, nanoparticles, nanotubes, and nanocrystals $-65.80 .+\mathrm{n}$ Thermal properties of small particles, nanocrystals, nanotubes

\section{Introduction}

The properties of small metal clusters have enjoyed a large interest over the past years. Their technical application in catalysis stems from the large surface-to-volume ratio while their properties differ from those of the bulk material raising the fundamental question about the statistical mechanics of finite systems.

The melting process of small clusters has early on been identified as the onset of isomer fluctuations [1,2. More recent investigations on $\mathrm{Ni}_{13-x} \mathrm{Al}_{x}$ alloy clusters in the microcanonical ensemble [3] show the relation between isomer fluctuations and the increase in entropy across the melting transition. The onset of the melting transition is marked by the fluctuations into the lowest energy isomer configurations which are measured by the root mean square bond length fluctuations [4 sometimes referred to as the Berry parameter $[5$. The phase space occupied by those fluctuations is small for small clusters resulting in a maximum in the specific heat at somewhat higher temperatures. The maximum in the specific heat in turn is determined by the onset of isomer fluctuations occupying a sufficiently large phase space fraction. A detailed overview of the increase of phase space with increasing particle number and the classification of isomers in terms of potential energy surfaces is given in Ref. [6]. Molecular Dynamics (MD) investigations [7] of the melting transition of larger $\mathrm{Au}_{N}$ clusters with $100<N<1000$ show that the bulk limit is gradually attained in agreement with experimental 8 findings.
Recently the empirical investigation of the melting of small Sn [9] and Ga 10] clusters has revealed a possible stability of the solid phase of the particles beyond the melting temperature of the bulk material. This result was interpreted as a consequence of the specific rigid ground state structure of the clusters and found support in microcanonical MD calculations for $\mathrm{C}, \mathrm{Si}, \mathrm{Ge}$, and $\mathrm{Sn}$ clusters [11] as well as for isokinetic $\mathrm{MD}$ investigations of $\mathrm{Sn}_{10}$ particles [12].

In metals the contribution from the conduction electrons to the binding energy has to be modelled by manybody potentials 13 14, which are numerically more involved than the thoroughly investigated Lennard-Jones systems 6 15 16. A prominent example is the Gupta potential (GP) [13, which can be derived in the second moment approximation from a tight binding model [17 and which correctly describes the surface contraction observed in metals:

$$
V\left(\left\{r_{i j}\right\}\right)=\sum_{i}^{N}\left[\sum_{j \neq i}^{N} A e^{-p \bar{r}_{i j}}-\sqrt{\sum_{j \neq i} \xi^{2} e^{-2 q \bar{r}_{i j}}}\right] .
$$

Here $N$ is the number of atoms, $i$ and $j$ are atom labels, $\bar{r}_{i j}=r_{i j} / r_{0}-1$, and $r_{i j}=\left|\boldsymbol{r}_{i}-\boldsymbol{r}_{j}\right|$ is the modulus of the distance between two atoms at positions $\boldsymbol{r}_{i}$ and $\boldsymbol{r}_{j}$. The parameters have been determined by fitting the experimental bulk lattice parameters and elastic moduli [18] as $A=0.1221 \mathrm{eV}, \xi=1.316 \mathrm{eV}, p=8.612$, and $q=2.516$ for Al. Distances are measured in units of the bulk first neighbour distance $r_{0}=2.864 \AA$. 
The present paper aims to shed light on how the melting transition evolves in the limit of small clusters. The method is a MC simulation in the canonical ensemble. A standard Metropolis algorithm is employed 19 20 with an update after each random displacement of an atom within an interval $\left[0, d_{\max }\right]$ in all spatial dimensions. $d_{\max }$ is set to yield an MC acceptance rate of 50 to $60 \%$. The resulting temperature dependence is roughly $d_{\max } \propto \sqrt{T}$. The boundary conditions are imposed by a hard wall cube with linear dimension $L$. Runs are performed with sampling rates $(\mathrm{SR})$ of up to $8 \times 10^{7}$ steps per temperature and atom. The fluctuations on the curves shown in the paper are a measure of the statistical error and appear near phase transition because of the usual critical slowing down. The ground state energies and configurations obtained within this method are in good agreement with earlier results 3 21]. The ground state configurations of the clusters discussed herein have the same symmetries as those of the 9-6 Sutton-Chen potentials [22.

An observable commonly studied in the context of melting transitions is the Berry parameter 4]

$$
\delta_{\mathrm{B}}=\frac{1}{N(N-1)} \sum_{i, j \neq i} \sqrt{\left\langle r_{i j}^{2}\right\rangle-\left\langle r_{i j}\right\rangle^{2}}\left\langle r_{i j}\right\rangle^{-1},
$$

where the brackets denote the thermodynamic average in the canonical ensemble. The parameter Eq. (2) measures the root mean square of the distance between two atoms averaged over all pairs. Even short isomer fluctuations with a subsequent return to the ground state can leave the cluster reordered, i.e., a previously nearest neighbour pair $r_{i j}$ may become a second- or third-neighbour pair after the fluctuation. Such a reordering leads to a notable increase in $\delta_{\mathrm{B}}$ allowing for the clear identification of a melting transition. Note that the Lindemann criterion of melting, which measures the atomic fluctuations with respect to their equilibrium positions, is usually employed for bulk systems but is less well suited for cluster systems 5 .

The second observable of interest is the specific heat, which can be obtained from the thermodynamic averages of the potential energy $V$ and its square:

$$
\frac{C}{k_{\mathrm{B}}}=\frac{1}{N k_{\mathrm{B}}^{2} T^{2}}\left(\left\langle V^{2}\right\rangle-\langle V\rangle^{2}\right)+\frac{3}{2} .
$$

Since the atoms are treated as classical particles the contribution from the kinetic energy is $C_{\text {kin }}=3 / 2 k_{\mathrm{B}}$ per atom.

In order to obtain a more intuitive understanding of the melting process a real-time visualisation of the simulation has been implemented. Figure 1 illustrates snapshots of an $\mathrm{Al}_{13}$ cluster in a volume of $\left(6 r_{0}\right)^{3}$ at temperatures (a) $T=0$, (b) $k_{\mathrm{B}} T=0.1 \mathrm{eV}$, and (c) $k_{\mathrm{B}} T=0.4$ $\mathrm{eV}$ corresponding to a solid, liquid, and dissociated cluster, respectively. The graphs show the corresponding normalised pair distribution functions $g(r)$ (arbitrary units). The ground state is icosahedral which is different but close to the slightly distorted icosahedral configuration obtained by first principle calculations 23. Contacts of the clusters

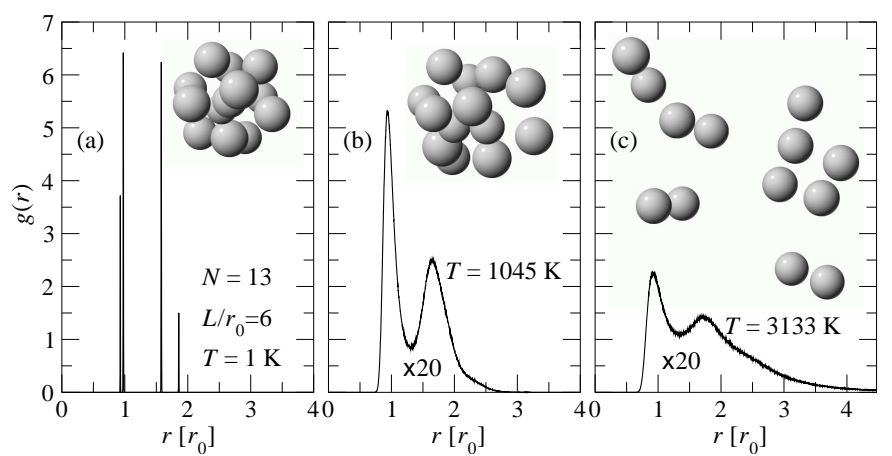

Fig. 1. Normalised pair distribution functions and snapshots of an $\mathrm{Al}_{13}$ cluster in a volume of $\left(6 r_{0}\right)^{3}$ at temperatures (a) $k_{\mathrm{B}} T=10^{-4} \mathrm{eV}$, (b) $k_{\mathrm{B}} T=0.09 \mathrm{eV}$, and (c) $k_{\mathrm{B}} T=0.27$ $\mathrm{eV}$ corresponding to a solid, liquid, and dissociated cluster, respectively. The pair distribution functions in panel (b) and (c) are enhanced by a factor of 20 for better visibility.

with the walls are rare events and the pressure is negligible in the solid and liquid phases for sufficiently large volumes, e.g., $L>4 r_{0}$ for $N=13$.

\section{Appearance of the melting transition}

A dimer of bound atoms cannot melt, only dissociate. Bulk metals show a well defined first order transition between their solid and liquid phases. This phase transition is accompanied by a divergence in the temperature dependence of the specific heat indicating the increase in entropy and the associated latent heat as well as by a discontinuous jump in the Berry parameter Eq. (2). Figure 2 shows how both signatures evolve [specific heat (a) and Berry parameter (b)] as the particle number is increased from 2 to 10 atoms.

$\mathrm{Al}_{5}$ is the smallest cluster with inequivalent bonds in its ground state configuration resulting in the abrupt increase in $\delta_{\mathrm{B}}$ once isomer fluctuations set in at around $k_{\mathrm{B}} T \sim 0.013 \mathrm{eV}$ or $T \sim 150 \mathrm{~K}$. For $\mathrm{Al}_{N}$ with $N \geq 6$ the increase in entropy is sufficiently large around a specific temperature to lead to a local maximum in the specific heat. As observed previously 3, the isomer fluctuations leading to the jump in $\delta_{\mathrm{B}}$ occur at lower temperatures than the maximum in the specific heat. The temperature of the discontinuity in $\delta_{\mathrm{B}}$ depends on the energy barrier between the ground state configuration and the lowest energy isomers [6].

Note that the presence of well defined signatures of the melting transition in the specific heat for clusters with $N<10$ is somewhat unexpected since the experimentally investigated $\mathrm{Na}_{N}$ clusters 24] appear to show no feature in the caloric curves for $N<100$ and for Lennard-Jones clusters [16] the corresponding signature disappears for $N<30$.

It is remarkable that the absence of signatures of a well defined melting transition does not imply that the clusters remain solid up to higher temperatures. For example, the real time visualisation of an $\mathrm{Al}_{4}$ particle (within the MC/GP approach, see Fig. 11for an illustration) at $T=0.1$ 
Ralph Werner: Melting and evaporation transitions in small Al clusters: canonical Monte-Carlo simulations

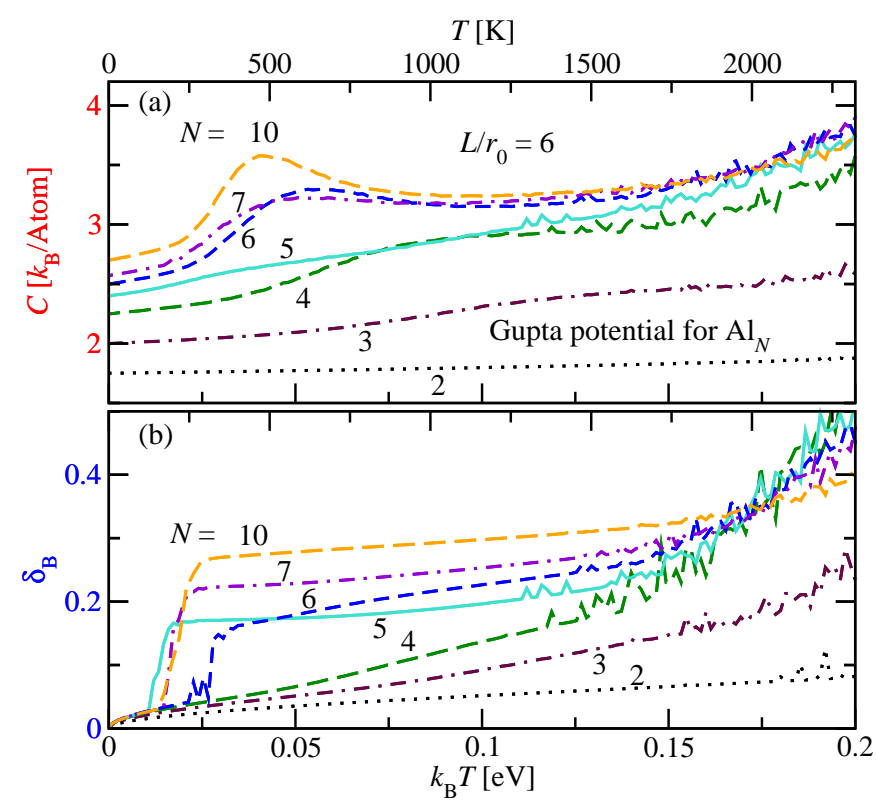

Fig. 2. Temperature dependence of the specific heat (a) and the Berry parameter (b) of $A l_{N}$ clusters with $N=$ $2,3,4,5,6,7,10$. A clear signature of a melting transition is observed for $N \geq 6$ in the specific heat and for $N \geq 5$ in the $\delta_{\mathrm{B}} . S R: 4 \times 10^{7}$ steps per temperature and atom.

$\mathrm{eV} / k_{\mathrm{B}} \sim 1160 \mathrm{~K}$ reveals that the cluster fluctuates out of its tetrahedral ground state into almost planar configurations and certainly cannot be considered solid. The small size of the phase space is responsible for the absence of a signature of the melting in the specific heat while the equivalence of all bonds in the ground state configuration result in a featureless $\delta_{\mathrm{B}}$.

In this sense all clusters investigated here with $N \geq 4$ are in a liquid state already at temperatures below the bulk melting temperatures of $\mathrm{Al}$ with $T_{\text {bulk }}=933 \mathrm{~K} \sim$ $0.08 \mathrm{eV} / k_{\mathrm{B}}$. For $N=2$ it is not possible to distinguish between a liquid and a solid phase. For $N=3$ the triangular structure is stable against fluctuations into collinear isomers up to $k_{\mathrm{B}} T \approx 0.15 \mathrm{eV}$.

The critical value of the Berry parameter at the melting transition $[5]$ was determined as $\delta_{\mathrm{B}} \sim 0.03-0.05$, which is referred to as the modified Lindemann criterion. These numbers are consistent with the present observation for clusters with $N \geq 5$, where at the melting transition a jump occurs from values of $\delta_{\mathrm{B}} \sim 0.03-0.05$ to values of $\delta_{\mathrm{B}}>1.5$ [1,2]20,25]. For smaller clusters with $N=3$ and 4 the temperature dependence of the Berry parameter is featureless. For $N=4$ at $T=0.1 \mathrm{eV} / k_{\mathrm{B}}$ and for $N=3$ at $T=0.15 \mathrm{eV} / k_{\mathrm{B}}$ the real time rendering of these clusters shows that they cannot be considered solid any more. At those temperatures the Berry parameter is $\delta_{\mathrm{B}} \approx 0.1$. These findings suggest that a more general sufficient criterion for clusters of all sizes not to be considered solid any more is $\delta_{\mathrm{B}} \geq 0.1$. The latter is close to the value given for the Lindemann criterion $\underline{5}$.

Multi-step melting [25 26] and isomer fluctuations [12] involving reordered atomic arrangements in the cluster are also consistent with that criterion since in both cases at

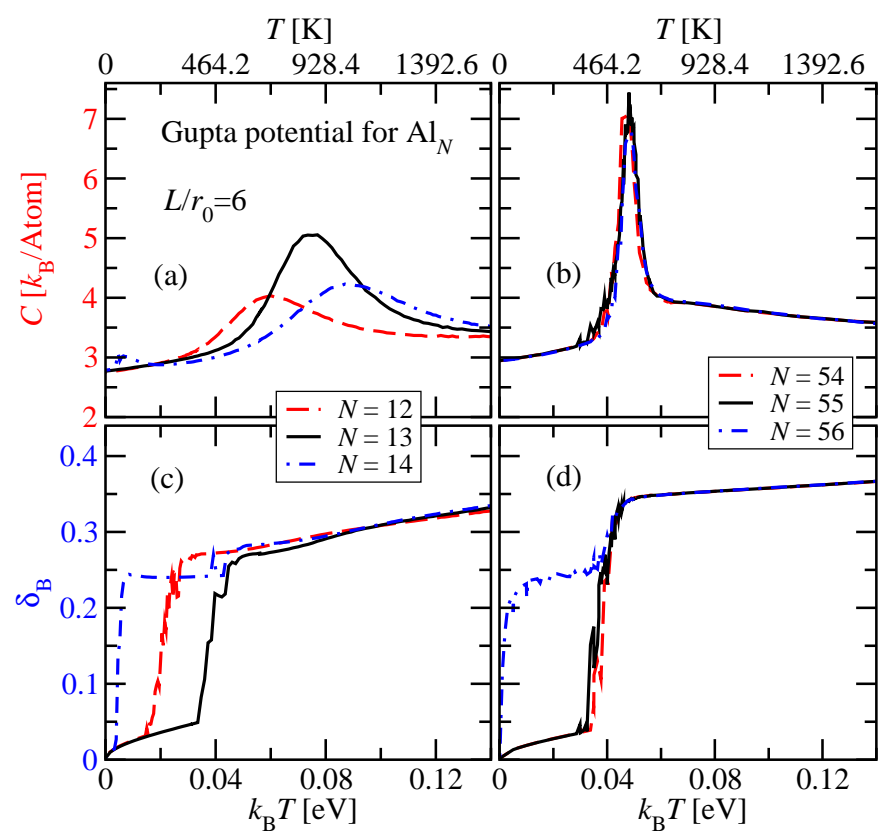

Fig. 3. Temperature dependence of the melting transition for clusters of size $N=12,13,14$ in (a) $+(c)$ and $N=54,55,56$ in $(b)+(d)$. (a) and (b): specific heat. (c) and (d): Berry parameter. Volume $L^{3} / r_{0}^{3}=6^{3}$. SR: 4 to $8 \times 10^{7}$ steps $[(a)+(c)]$ and 1 to $2 \times 10^{7}$ steps $[(b)+(d)]$ per temperature and atom.

least a group of atoms does not remain located at their ground state positions when $\delta_{\mathrm{B}} \geq 0.1$.

Both specific heat and Berry parameter in Fig. (2) show an increase for temperatures above $\sim 1800 \mathrm{~K}$ accompanied by an increase in fluctuations due to statistical errors. As will be discussed further below in detail these are signatures of the dissociation transition.

\section{Closed-shell effects}

The influence of closed shells on the cohesive energies of metal clusters [22, 23, 21, 6] and their melting points [8, [25 20] has been a focus of research for quite some time. A closed-shell cluster has a large gap to the first excited isomer while adding or removing an atom leads to a number of degenerate ground state configurations separated by a potential barrier. This manifests itself in a smoother specific heat anomaly as well as in a jump in the Berry parameter at much lower temperatures as compared to the closed-shell counterpart. This is shown in Fig. 3(a) and (c) for the sets of $N=12,13,14$ and in Fig. B(b) and (d) for the sets of $N=54,55,56$ atoms. The upper panels (a) and (b) show the specific heat, the lower panels (c) and (d) the Berry parameter.

Notably the 14 and 56 atom clusters have a very low barrier between isomers. The real time visualisation reveals that these fluctuations occur in $\mathrm{Al}_{14}$ not only by jumps of the $14^{\text {th }}$ atom on the surface of the $\mathrm{Al}_{13}$ icosahedron but by absorption of the $14^{\text {th }}$ atom into the outer shell and simultaneous pushing of another atom onto the surface. In $\mathrm{Al}_{56}$ the $56^{\text {th }}$ atom is absorbed into the outer 
Ralph Werner: Melting and evaporation transitions in small Al clusters: canonical Monte-Carlo simulations

shell even in the ground state configuration (see SuttonChen 9-6 in Ref. [22]). This leads to the large jump in $\delta_{\mathrm{B}}$ at very low temperatures [dash-dotted lines in Fig. B(c) and (d)]. In $\mathrm{Al}_{14}$ the phase space is sufficiently large to induce an anomaly in the specific heat [dash-dotted line in Fig. [3(a)] at the same temperature. Together with the main maximum at higher temperatures this may be referred to as a two-step melting mechanism 25, 26, 27.

The narrower specific heat anomaly at the melting transition and the smaller discrepancy between the maximum of the specific heat and the jump in the Berry parameter for the larger systems in Fig. B(b) and (d) illustrate how the thermodynamic limit is gradually approached as the cluster size is increased 7,25$] 28$. Note that for larger clusters less sampling steps per temperature and atom are required to obtain smooth curves. The larger phase space of the larger clusters [6] results in the better convergence of the observables.

The canonical ensemble as shown for $\mathrm{Al}_{13}$ in Fig. 3(a) and (b) yields a somewhat lower specific heat 25] and an onset of the isomer fluctuations at lower temperatures as compared with the results for the microcanonical ensemble in Ref. [3]. The discrepancies can be attributed to the energy fluctuations in the canonical ensemble that allow the potential barriers between different isomers to be overcome at lower temperatures.

\section{Ideal gas limit}

The well defined high temperature limit, where the system has the properties of an ideal gas, yields a test of the numerical methods and is essential for determining the latent heat of the evaporation transition in Sec. 15 Figure 4 illustrates the large $T$ behaviour of the specific heat (full line, right scale) and the Berry parameter (broken line, left scale) for $\mathrm{Al}_{2}$ (a), $\mathrm{Al}_{7}$ (b), and $\mathrm{Al}_{13}$ (c). Since the Boltzmann weight can be expanded as $\exp \left\{-V /\left(k_{\mathrm{B}} T\right)\right\}=$ $1-V /\left(k_{\mathrm{B}} T\right)+\mathrm{O}\left(T^{-2}\right)$, the specific heat attains the limit as $C=\frac{3}{2} k_{\mathrm{B}}+a T^{-3}+\mathrm{O}\left(T^{-4}\right)$ while the Berry parameter is $\delta_{\mathrm{B}}=\delta_{\infty}+b T^{-1}+\mathrm{O}\left(T^{-2}\right)$ with $\delta_{\infty}=0.3768(1)$. Here $a$ and $b$ are volume and particle number specific constants. An analytical scaling analysis reveals that $\delta_{\infty}$ depends on the container geometry but neither on its volume nor the particle number.

\section{Dissociation}

Between the low temperature liquid phase and the high temperature ideal gas limit Fig. 4] shows clear maxima both in the specific heat and the Berry parameter for $\mathrm{Al}_{2}$, $\mathrm{Al}_{7}$, as well as for $\mathrm{Al}_{13}$. The feature is generic for all cluster sizes and can be associated with the dissociation transition. The dissociation anomaly in the specific heat stems from the increase in entropy across the dissociation transition while the Berry parameter is enhanced through the short-time elongation and return of an atom from and to the cluster. The latter involves an energy fluctuation and

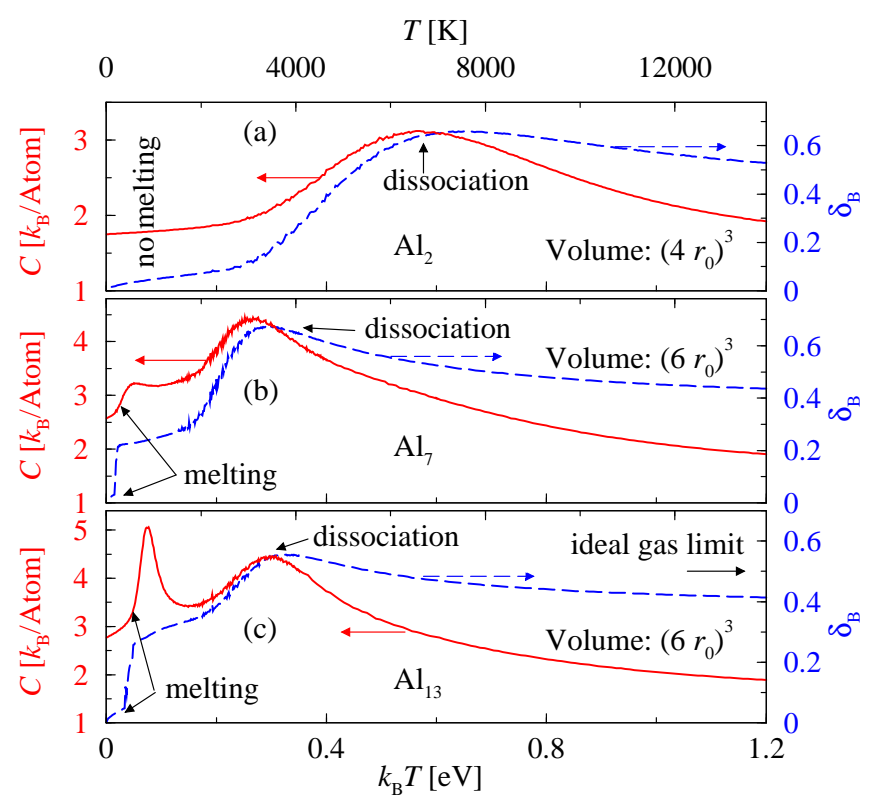

Fig. 4. Temperature dependence of the specific heat (full line, left scale) and the Berry parameter (broken line, right scale) of a 2 (a), 7 (b), and 13 (c) atom Al cluster. The dissociation anomaly is always present while the signature of the melting transition evolves with system size. SR: $4 \times 10^{7}[(\mathrm{a})+(\mathrm{b})]$ and $2 \times 10^{7}$ (c) steps per temperature and atom.

is consequently suppressed in a microcanonical or isokinetic ensemble.

Figure 5 shows the container size dependence of the dissociation transition of an $\mathrm{Al}_{13}$ cluster for volumes of $L^{3}=\left(4 r_{0}\right)^{3},\left(6 r_{0}\right)^{3},\left(10 r_{0}\right)^{3},\left(15 r_{0}\right)^{3}$, and $\left(20 r_{0}\right)^{3}$. For $L^{3}=\left(4 r_{0}\right)^{3}$ the density $N / L^{3}=0.0144$ mole $/ \mathrm{cm}^{3}$ is only a factor 7 smaller than that of bulk Al with $0.1 \mathrm{~mole} / \mathrm{cm}^{3}$. This leads essentially to a suppression of the evaporation transition, which is replaced by a smooth crossover (see also Fig. 6). Note that even macroscopic particles do not exhibit a sharp evaporation transition in a finite, constant volume imposing a finite, temperature dependent gas-phase partial pressure. As a consequence there is a liquid-vapour coexistence region which is given by the width of the specific heat anomalies shown in Fig. [5 The snapshot shown in Fig. [7(b) in Sec. [6] is taken in the liquidvapour coexistence region of $\mathrm{Al}_{13}$ for $L^{3}=\left(20 r_{0}\right)^{3}$, where liquid fragments of the cluster coexist with evaporated single atoms.

Both the anomaly in the specific heat (upper panel in Fig. (5) and the Berry parameter (lower panel in Fig. (5) increase with increasing volume and become narrower. In an infinite volume the partial pressure of the gas phase is zero and both quantities are expected to exhibit a sharp peak at the transition. Note that the fluctuations of the graphs in Fig. [5increase with increasing volume as a consequence of the enlarged phase space and thus limit the simulations to small volumes. The melting transition is independent of the container volume. This is expected since the pressure in the system is essentially zero when all atoms are condensed [29]. 
Ralph Werner: Melting and evaporation transitions in small Al clusters: canonical Monte-Carlo simulations

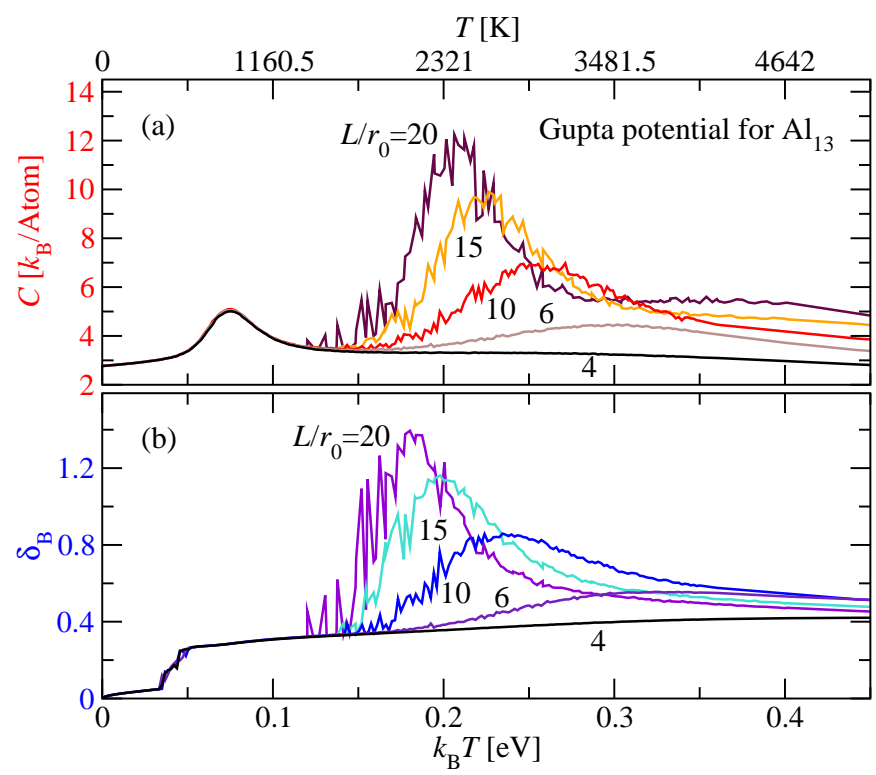

Fig. 5. Sample volume dependence of the dissociation transition for four different sampling volumes of $L^{3} / r_{0}^{3}=4^{3}, 6^{3}, 10^{3}$, $15^{3}, 20^{3}$. Both the anomaly in the specific heat (upper panel) and the Berry parameter (lower panel) increase with increasing volume as a consequence of the increased phase space. SR: $2 \times 10^{7}\left(L^{3} / r_{0}^{3}=4^{3}, 6^{3}, 10^{3}\right)$ and $4 \times 10^{7}\left(L^{3} / r_{0}^{3}=15^{3}, 20^{3}\right)$ steps per temperature and atom.

For sufficiently small densities or, equivalently, large volumes the latent heat of the melting transition is much smaller than the energy released in the evaporation transition 29. For $\mathrm{Al}_{13}$ this becomes apparent from the caloric curves of the total enclosed system shown in Fig. [6] which are obtained by simply integrating the specific heat (Fig. 5. for a fixed volume, i.e., $E_{V}(T)=\int_{0}^{T} C\left(T^{\prime}\right) d T^{\prime}-E_{\mathrm{B}}$. The binding energy per atom for $N=13$ is $E_{\mathrm{B}}=2.60088 \mathrm{eV}$. Since the transitions are smeared out the determination of the latent heat is somewhat ambiguous. Extrapolation of the linear segments of the caloric curves above and below the melting transition (dashed lines in Fig. 6) and reading off the energy difference at the temperature of the specific heat maximum yields $\Delta E_{\text {melt }} \approx 0.08 \mathrm{eV}$.

The evaporation transition is very broad for the volumes (densities) under investigation and the ideal gas limit $\left(E_{\text {ideal }}(T)=2 / 3 k_{\mathrm{B}} T\right.$, dash-dotted line in Fig. 6) is only attained for $k_{\mathrm{B}} T>1 \mathrm{eV}$ even for $L / r_{0}=20$. For $L / r_{0}=4$ the ideal gas limit is reached only for temperatures much larger than the binding energy per atom, i.e., $k_{\mathrm{B}} T \gg E_{\mathrm{B}}$. Consequently the total latent heat of the evaporation can only be given approximately as $\Delta E_{\text {evap }} \approx$ $2.26 \mathrm{eV}$, which corresponds satisfactorily to the potential energy expectation value $\langle V\rangle\left(T_{\text {evap }}\right)=2.35 \mathrm{eV}$ near the onset of the evaporation transition at $k_{\mathrm{B}} T_{\text {evap }}=0.15 \mathrm{eV}$.

Results for $\mathrm{Al}_{55}$ (not shown) are similar with slightly narrower evaporation anomalies compared with those of $\mathrm{Al}_{13}$ with comparable densities $N / L^{3}$. Closed shell effects do not play an observable role in the evaporation transition.

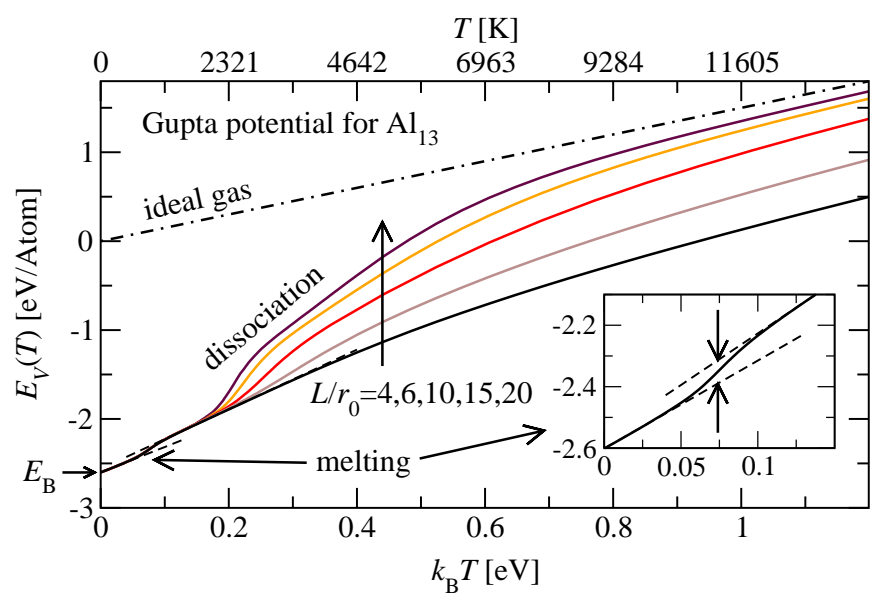

Fig. 6. Caloric curves as obtained from integrating the specific heat data in Fig. 15 for sampling volumes of $L^{3} / r_{0}^{3}=4^{3}, 6^{3}, 10^{3}$, $15^{3}, 20^{3}$. The inset shows the determination of the latent heat $\Delta E_{\text {melt }} \approx 0.08 \mathrm{eV}$ of the melting transition. The dash-dotted line is the ideal gas case reached only for temperatures much larger than the binding energy, i.e., $k_{\mathrm{B}} T \gg 2.60088 \mathrm{eV}$.

From the above discussion follows that the dissociation anomalies in the specific heat usually dominate in size over those of the melting transition especially for clusters that do not have closed-shell structures. An example is $\mathrm{Al}_{7}$ as

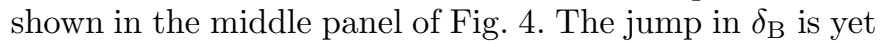
well pronounced confirming its sensitivity to the melting transition [5].

\section{Fragmentation}

On closer examination the curves of the specific heat of $\mathrm{Al}_{13}$ for sufficiently large volumes show another broad local maximum at higher temperatures. For $L / r_{0}=20$ as shown in Fig. [5 (a) this maximum is found near $k_{\mathrm{B}} T \sim 0.36$ $\mathrm{eV}$. This effect is readily explained by the fragmentation of the cluster into dimers and trimers at temperatures above the onset of the evaporation transition near $k_{\mathrm{B}} T_{\text {evap }}=0.15 \mathrm{eV}$. The dimers and trimers in turn dissociate at temperatures near the second local maximum. For smaller volumes (larger densities) the effect is not visible because the signatures become very broad.

To quantify the effect, Fig. [7(a) shows the maxima of the evaporation anomaly of the specific heat as a function of the cluster size at constant densities of $\rho=N / L^{3}=$ $1.625 \times 10^{-3} r_{0}^{-3}$, which correspond for $N=13$ to $L / r_{0}=$ 20 as shown in Fig. 5(a). The temperature of the specific heat maximum increases with increasing size for $N \geq 6$ but for $N \leq 6$ shows a non-monotonous decrease with increasing cluster size. In other words, especially the dimers and trimers are more stable with respect to larger clusters. The superposition of their specific heat maxima at $k_{\mathrm{B}} T_{\max }=0.415(5) \mathrm{eV}$ and $k_{\mathrm{B}} T_{\max }=0.275(5) \mathrm{eV}$ for the dimer and trimer, respectively, leads to the second maximum of the curve for $L / r_{0}=20$ in Fig. [5 (a). Fig. [7(b) shows a snapshot of $\mathrm{Al}_{13}$ at $k_{\mathrm{B}} T=0.25 \mathrm{eV}$ with $L / r_{0}=20$, where a dissociated single atom, two dimers 


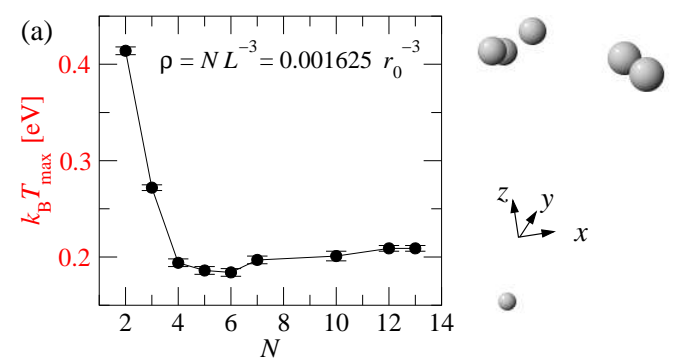

(b)

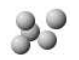

Fig. 7. (a) Temperature of the maximum of the specific heat at the evaporation anomaly as a function of the cluster size at a constant density of $\rho=1.625 \times 10^{-3} r_{0}^{-3}$. The line is a guide to the eye. The minimum at $N=6$ implies that those clusters dissociate first while trimers and dimers are stable to much higher temperatures. Larger clusters dissociate predominantly into dimers and trimers at intermediate temperatures as shown by the snapshot in panel (b) for $A l_{13}$ at $k_{\mathrm{B}} T=0.25 \mathrm{eV} . \mathrm{SR}$ for the determination of $T_{\max }: 10^{8}$ steps per temperature and atom.

and a trimers are visible together with a (liquid) pentamere. Qualitatively comparable results are found for $\mathrm{Al}_{55}$ (not shown) with $k_{\mathrm{B}} T_{\max }=0.23(1) \mathrm{eV}$ at similar densities.

Note that the stability of the dimers and trimers towards dissociation is an entropic effect since the binding energy per atom decreases monotonously with decreasing size for $N \leq 1330$. Consequently the observed presence of dimers and trimers is not in contradiction with the results from density functional theory calculations [31, which find the single atom emission to be energetically the dominant dissociation channel. The dimers and trimers are at least in part formed by fusion of single evaporated atoms. On the other hand, this fragmentation behaviour is not directly transferable to experiments on Al clusters because the Gupta potentials do not yield the correct planar structure for $N \leq 5[23,31$.

\section{Conclusions}

The results from the Monte-Carlo simulation of $\mathrm{Al}_{N}$ clusters modelled with many-body GPs reveals the appearance of a distinct feature of the melting transition in the specific heat and the Berry parameter for $N \geq 6$. The energy fluctuations in the canonical ensemble lead to an onset of isomer fluctuations at lower temperatures than in the microcanonical ensemble. $\mathrm{Al}_{N}$ clusters with $N \geq 4$ are liquid at the bulk melting temperature. The present analysis suggests that a generalised sufficient criterion for clusters not to be considered solid any more is a Berry parameter of $\delta_{\mathrm{B}} \geq 0.1$. Larger clusters with closed-shell configurations exhibit sharper signatures of the melting transition than others.

For higher temperatures clusters of all sizes undergo a dissociation transition which is accompanied by container volume dependent anomalies both in the specific heat and Berry parameter. Dimers and trimers are more stable towards dissociation than larger clusters. The details of the features depend on the potential but the qualitative results are generic. For example, MC simulations with GPs and parameters for $\mathrm{Au}$ yield qualitatively very similar results.

The author thanks M. Blom, P. Schmitteckert, G. Schneider, D. Schooss, M. Vojta, and P. Wölfle for instructive discussions. The work was supported by the Center for Functional Nanostructures of the Deutsche Forschungsgemeinschaft within project D1.5.

\section{References}

1. F. G. Amar and R. S. Berry, J. Chem. Phys. 85, 5943 (1986).

2. J. Jellinek, T. L. Beck, and R. S. Berry, J. Chem. Phys. 84, 2783 (1986).

3. E. B. Krissinel and J. Jellinek, Int. J. Quant. Chem. 62, 185 (1997).

4. R. S. Berry, T. L. Beck, H. L. Davis, and J. Jellinek, Adv. Chem. Phys. 70B, 75 (1988).

5. Y. Zhou, M. Karplus, K. D. Ball, and R. S. Berry, J. Chem. Phys. 116, 2323 (2002).

6. D. J. Wales et al., Adv. Chem. Phys. 115, 1 (2000).

7. F. Ercolessi, W. Andreoni, and E. Tosatti, Phys. Rev. Lett. 66, 911 (1991).

8. M. Y. Efremov et al., Phys. Rev. Lett. 85, 3560 (2000).

9. A. A. Shvartsburg and M. F. Jarrold, Phys. Rev. Lett. 85, $2530(2000)$.

10. G. A. Breaux et al., Phys. Rev. Lett. 91, 215508 (2003).

11. Z.-Y. Lu, C.-Z. Wang, and K.-M. Ho, Phys. Rev. B 61, 2329 (2000).

12. K. Joshi, D. G. Kanhere, and S. A. Blundell, Phys. Rev. B 66, 155329 (2002).

13. R. P. Gupta, Phys. Rev. B 23, 6265 (1981).

14. D. Tománek, S. Mukherjee, and K. H. Bennemann, Phys. Rev. B 28, 665 (1983).

15. J. D. Honeycutt and H. C. Andersen, J. Phys. C 91, 4950 (1987).

16. D. D. Frantz, J. Chem. Phys. 115, 6157 (2001).

17. W. Zhong, Y. S. Li, and D. Tománek, Phys. Rev. B 44, 13053 (1991).

18. F. Cleri and V. Rosato, Phys. Rev. B 48, 22 (1993).

19. M. P. Allen and D. J. Tildesley, Computer Simulation of Liquids, Oxford Science Publications (Clarendon Press, Oxford, 1989).

20. J. Wanga et al., Solid State Commun. 119, 13 (2001).

21. G. W. Turner, R. L. Johnston, and N. T. Wilson, J. Chem. Phys. 112, 4773 (2000).

22. J. P. K. Doye and D. J. Wales, New. J. Chem. 1998, 733 (1998).

23. R. Ahlrichs and S. D. Elliot, Phys. Chem. Chem. Phys. 1, 13 (1999).

24. M. Schmidt, J. Donges, T. Hippler, and H. Haberland, Phys. Rev. Lett. 90, 103401 (2003).

25. P. Blaise and S. A. Blundell, Phys. Rev. B 63, 235409 (2001).

26. R. E. Kunz and R. S. Berry, Phys. Rev. E 49, 1895 (1994).

27. H.-P. Cheng and R. S. Berry, Phys. Rev. A 45, 7969 (1992).

28. M. Schmidt et al., Phys. Rev. Lett. 79, 99 (1997).

29. M. Schmidt et al., Phys. Rev. Lett. 87, 203402 (2001).

30. Binding energies for $\mathrm{Al}_{N}$ using Gupta potentials are $E_{\mathrm{B}}=$ $1.4955,1.8331,2.0649,2.1828,2.2957,2.3528$, and 2.4725 $\mathrm{eV} /$ Atom for $N=2,3,4,5,6,7$, and 10, respectively.

31. B. K. Rao and P. Jena, J. Chem. Phys. 111, 1890 (1999). 